\title{
Ecología trófica y reproductiva de Hemibrycon sierraensis (Characiformes: Characidae), pez endémico del río Gaira, Sierra Nevada de Santa Marta, Colombia
}

\author{
Eduardo J. Ibarra-Trujillo \& Carlos A. García-Alzate* \\ Programa de Biología, Colecciones Científicas, Museo de Biología, Facultad de Ciencias Básicas, Universidad del \\ Atlántico, Barranquilla, Colombia; ibarraeduardojunior@gmail.com, carlosgarciaa@mail.uniatlantico.edu.co \\ * Correspondencia
}

Recibido 21-II-2017. Corregido 10-IV-2017. Aceptado 10-V-2017.

\begin{abstract}
Trophic and reproductive ecology of Hemibrycon sierraensis (Characiformes: Characidae), endemic fish of Gaira River, Sierra Nevada of Santa Marta, Colombia. The study of feeding habits in fishes can give important information about their role in the fish community, their functional role in the ecosystem, and their relationships with all available resources. We studied H. sierraensis from Gaira River and performed eight bi-monthly samples between May 2014 and November 2015. We analyzed fish stomach contents by the numerical $(\% \mathrm{~N})$, volume $(\% \mathrm{~V})$ and frequency of occurrence $(\% \mathrm{FO})$ methods; we also calculated the index of food importance (IA), coefficient of emptiness (CV), trophic niche breadth (used H') and condition factor (K). Additionally, we determined the sex ratio, gonadosomatic index (RGS), fecundity, minimum and average size at sexual maturity, and oocyte diameter by standard methods. The Gaira River has irregular meanders with clear waters, and high current velocity through its drainage; it is generally oligotrophic, with a tendency to eutrophic condition in some places. Observed dissolved oxygen values were high $(5.4 \mathrm{mg} / \mathrm{L})$, electrical conductivity was $68.63 \mu \mathrm{S} / \mathrm{cm}$, and $\mathrm{pH}$ was found neutral. We analyzed stomach contents of 477 specimens, and found a $1.59 \%$ coefficient of vacuity. The species was found euryphagic in its feeding habits $\left(\mathrm{H}^{\prime}=0.69\right)$, omnivorous tending to insectivorous; its diet consisted mainly of Trichoptera larvae (Atanatolica sp.), followed in importance by snails (Gastropoda Pomacea sp.) and dipteran larvae such as Simulium sp. Significant differences in both frequency of occurrence and the volume consumed were observed for the different size classes, as well as between different seasons of the year. The condition factor indicated that the species was well fed. Sex was determined for 424 fish, of which 241 were male and 183 female, given a ratio of 1.3: 1 (male-female) which resulted significantly different (Chi 2: 16.14; $\mathrm{p}=0.02$ ). The gonadosomatic index indicated that reproduction occurs during the high water river phase, fecundity was high, and oocyte diameter was relatively small. Males reached sexual maturity at an average size of $67.08 \mathrm{~mm} \mathrm{SL}$, and females at $70.68 \mathrm{~mm}$ SL. Their life history strategy is seasonal $\mathrm{r}$. Management and conservation plans for this species are required due to human intervention in their habitat, originated by the growing tourist activity in the area. Rev. Biol. Trop. 65 (3): 1033-1045. Epub 2017 September 01.
\end{abstract}

Key words: diet, reproduction, teleost, diversity, freshwater tropical fish.

Los pequeños cursos de agua de alta montaña representan una fuente hídrica para el abastecimiento urbano y rural, y se encuentran dotados de una fauna distintiva y poco conocida (Luiz, Agostinho, Gomes, \& Hahn, 1998; Bojsen \& Barriga, 2002; Román-Valencia, Ruiz, \& Giraldo, 2008). El interés por estudiar este tipo de ambientes ha aumentado en los últimos años, debido a la presión antrópica generada por el turismo acelerado, la agricultura y la pérdida de los bosques naturales, que ocasionan un deterioro en la calidad del recurso hídrico, la extinción de recursos naturales inexplorados y de especies regionales, muchas de ellas aún no descritas y posiblemente de gran importancia económica y/o ecológica (García-Alzate, Román-Valencia, \& Barrero, 2012). En la Sierra Nevada de Santa Marta nacen 34 cuencas 
hidrográficas principales que abastecen las poblaciones de los departamentos del Magdalena, Cesar y Guajira, el conocimiento sobre la ictiofauna allí presente es pobre y en muchos casos se limita a listados de especies, situación que hace necesario el desarrollo de estudios ecomorfológicos, de ecología trófica y reproductiva, con el fin de proporcionar herramientas para la conservación de las mismas (Lasso, Sánchez-Duarte, \& Blanco-Libreros, 2015).

El estudio de los hábitos alimenticios de las especies es importante, ya que permite evaluar su estado en la comunidad (nivel trófico) y por lo tanto, el efecto que puede producirse en cualquier tipo de uso y gestión de la misma (explotación, manejo, control de la calidad de agua, ausencia de presas, ausencia de depredadores), así mismo, es fundamental para entender las estrategias de alimentación (comportamiento selectivo u oportunista) y la dinámica dentro del nicho (Bellocq, 2000). Esta última representa una integración de varios componentes ecológicos que incluyen comportamiento, condición y uso de hábitat, toma de energía y las interacciones interespecíficas e intraespecíficas.

El género Hemibrycon es un representante de la familia de peces Characidae que comprende 36 especies válidas y de estas, 21 se distribuyen en los ríos de Colombia (García-Alzate, Román-Valencia, \& Taphorn, 2015). Del total, solo se ha publicado estudios del hábitat, dieta y reproducción para $H$. quindos (Román-Valencia \& Botero, 2006), H. boquiae (Román-Valencia et al., 2008) y H. brevispini (Román-P., Román-Valencia, \& Taphorn, 2014), pero es evidente la falta de información de referencia para la conservación de las mismas. Por su parte, $H$. sierraensis se distribuye en la parte media del río Gaira en la Sierra Nevada de Santa Marta; es una especie endémica y fue recientemente descrita, por lo cual, se desconocen aspectos sobre su ecología, hábitos tróficos, reproductivos y ecosistémicos (García-Alzate et al., 2015). Por lo anterior, el objeto de este trabajo fue determinar aspectos de la ecología trófica y reproductiva de $H$. sierraensis pez endémico del río Gaira,
Sierra Nevada de Santa Marta, Vertiente Caribe, Colombia.

\section{MATERIAL Y MÉTODOS}

Área de estudio: El trabajo se realizó en el río Gaira, en el corregimiento de Minca a 670 m.s.n.m., ubicado al norte del departamento del Magdalena, en las estribaciones de la Sierra Nevada de Santa Marta (SNSM), Caribe, Colombia (118'32" N \& 747'7" W). Registra una temperatura anual promedio $24.5{ }^{\circ} \mathrm{C}$ y un patrón de distribución de la precipitación bimodal, con un período de altas lluvias desde mayo hasta noviembre, y bajas lluvias o sequía desde diciembre hasta abril. El promedio de precipitación mensual desde el 2004 hasta el 2014 fue de $207.1 \mathrm{~mm}$, octubre el más lluvioso con $470.3 \mathrm{~mm}$, y febrero el de menor registro con $0.3 \mathrm{~mm}$ (estación pluviométrica MINCA, Instituto de Hidrología, Meteorología y Estudios Ambientales de Colombia-IDEAM; Fig. 1).

Fase de campo: Se realizó un total de ocho muestreos entre mayo de 2014 y noviembre de 2015; se incluyó los períodos de bajas y altas lluvias para la zona. Las capturas de los peces se llevaron a cabo con red de arrastre de 2 $\mathrm{m}$ de largo, $0.50 \mathrm{~m}$ de altura, con un tamaño de ojo de malla $0.5 \mathrm{~cm}$, y atarraya $2 \mathrm{~m}$ de diámetro y $0.5 \mathrm{~cm}$ de ojo de malla. Los muestreos se efectuaron entre las 8:00 y las 17:00 horas, y se hizo barridos a favor y en contra de la corriente, y de ribera a ribera, con el fin de incluir todos los biotopos del río (se sigue a Morales \& García-Alzate, 2016). Los ejemplares capturados se colocaron en neveras de poliestireno con hielo, inmediatamente después de su captura, para evitar la digestión del contenido estomacal. Para cada individuo se evaluó el peso total (g) con una balanza analítica ADVENTURER PRO AV 264 hasta cuatro décimas de precisión $(0.0001 \mathrm{~g})$, y la longitud total y estándar (mm) con un calibrador digital hasta centésimas de milímetro. A continuación las muestras fueron disectadas con un corte uroventral para extraer estómagos, intestinos y gónadas. Cada una de estas estructuras fue pesada (en g), medida 


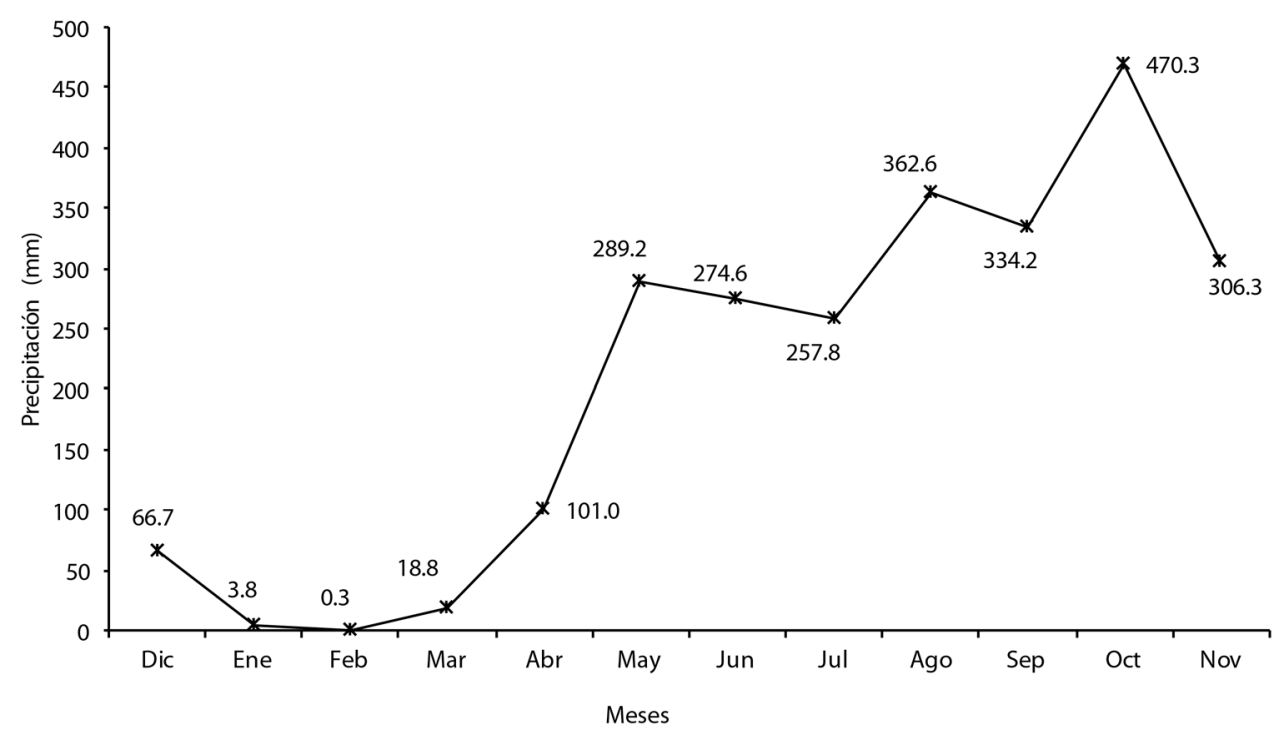

Fig. 1. Precipitación promedio mensual multianual, estación Minca, Sierra Nevada de Santa Marta, Caribe, Colombia (2004-2014).

Fig. 1. Multi-year monthly rainfall, Minca Station, Sierra Nevada of Santa Marta, Caribe, Colombia (2004-2014).

(en mm), fijadas en alcohol al $70 \%$ y depositadas en frascos plásticos debidamente rotulados con la información pertinente del ejemplar (número de etiqueta y de ejemplar, fecha y lugar de recolecta, sexo y estructura fijada) (se sigue a Morales \& García-Alzate, 2016).

Los ejemplares fueron etiquetados con un número, fecha, lugar y el nombre de recolectores; posteriormente, fueron fijados en formol al $10 \%$ y conservados en alcohol al $70 \%$ y depositados en la colección de peces del museo de colecciones biológicas de la Universidad del Atlántico, Barranquilla, Región Caribe, Colombia (UARC-IC). El total de ejemplares se distribuyeron en tallas según su longitud estándar (L.E.), al utilizar la ecuación: $A=N / R$, $\mathrm{R}=\sqrt{\mathrm{n}}$ y $\mathrm{N}=$ Max-Min, donde $\mathrm{A}=$ amplitud de los intervalos, $\mathrm{R}=$ número de intervalos, $\mathrm{n}=$ número de observaciones, Max=valor máximo, Min=valor mínimo. Además, se evaluaron algunas variables fisicoquímicas in situ como: $\mathrm{pH}$ con un potenciómetro LAB-KITS ELE- 711, temperaturas del aire y superficial del agua $\left({ }^{\circ} \mathrm{C}\right)$, oxígeno disuelto $(\mathrm{mg} / \mathrm{L})$ con un oxímetro digital LAB-KITS ELE-716, la conductividad $(\mu \mathrm{s} / \mathrm{cm})$ y salinidad $(\mathrm{mg} / \mathrm{L})$ con un conductímetro WEILHEIM Cond 3110.

Fase de laboratorio: Para evaluar la dieta se procedió a analizar el contenido del estómago y de la primera porción del intestino, se depositó en cajas Petri y se examinó en el microscopio. El contenido fue agrupado por grupos taxonómicos o categoría trófica, de acuerdo a su origen, fue identificado con ayuda de claves (Domínguez \& Fernández, 2009; Roldan, 1988; Streble \& Krauter, 1987). El contenido estomacal se analizó con el método numérico $(\% \mathrm{~N}) \mathrm{N}=(n i / N p)^{*} 100$, donde $n_{i}$ es el número total de representantes de la presa i; $\mathrm{N}_{\mathrm{p}}$ es el número total de presas consumidas, método volumétrico $(\% \mathrm{~V})$, al colocar una hoja de papel milimétrico de $10 \times 10$ cuadros, en el fondo de una caja Petri, el contenido fue depositado en la caja donde fue extendido y separados por ítems alimenticios; el número total de cuadros ocupados por los distintos ítems del mismo estómago, representó el $100 \%$ de la dieta de ese individuo y el método de frecuencia de ocurrencia $(\% \mathrm{FO})$ : FO $\%=\mathrm{Ei} / \mathrm{Et} * 100$, 
donde $\mathrm{E}_{\mathrm{i}}$ es el número de estómagos con la presa $\mathrm{i} ; \mathrm{E}_{\mathrm{t}}$ es el número total de estómagos examinados (Se sigue a Hyslop, 1980).

Para estimar las presas de mayor aporte en la dieta de la especie objeto de estudio, se aplicó el Índice de Importancia Alimentaria (I.A) por medio de la ecuación: IA $=(\% \mathrm{~V} \mathrm{x}$ $\%$ FO) $/ 100$, donde se relaciona el porcentaje del volumen $(\% \mathrm{~V})$ con la frecuencia $(\% \mathrm{FO})$ de cada ítem alimenticio (Lauzanne, 1975; Kawakami \& Vazzoler, 1980). Para comprobar la distribución de los datos, se realizó una prueba de bondad de ajustes (Shapiro Wilk) a los valores del número, volumen y frecuencia de ocurrencia de los ítems alimenticios. Seguidamente, se empleó Kruskal-Wallis (K-W) para observar la significancia estadística de las diferencias de la dieta consumida entre períodos climáticos, sexo y tallas por medio del programa Past 3.0 bajo Windows.

Se calculó la amplitud del nicho trófico a partir del Índice de diversidad de ShannonWeaver $\left(\mathrm{H}^{\prime}\right)$ (1949), por medio de la ecuación: $\mathrm{H}^{\prime}=(-\Sigma p i \mathrm{Ln} p i)$ donde, $p i$ es el número de individuos del iesimo componente trófico por el total de organismos de la muestra. La proporción de la diversidad observada se comparó con la máxima diversidad esperada por medio del índice de equidad de Pielou ( $\left.\mathrm{J}^{\prime}\right)$ (Krebs, 1998) $\mathrm{J}^{\prime}=\mathrm{H}^{\prime} / \mathrm{H}_{\text {max }}$; donde, $\mathrm{H}_{\max }$ es el logaritmo natural del número de componentes tróficos por muestra, cuyos valores tienen un rango de cero (0) a uno (1), cuando son menores a 0.6 el depredador se considera especialista, cercanos a 1 ó mayores de 0.6 su espectro es generalista. Se analizó el coeficiente de vacuidad (Va) (Hyslop, 1980), que permite conocer el período de alimentación de la especie, y se calculó al aplicar la ecuación $\mathrm{Va}=(\mathrm{n} / \mathrm{N}) * 100$, donde $\mathrm{n}=$ número de estómagos vacíos, $\mathrm{N}=$ Número de estómagos examinados. Así mismo se evaluó el factor de condición K (Vazzoler, 1996) para conocer el grado de robustez y desarrollo del pez, que presupone un incremento alométrico, del peso como función del tamaño del individuo al aplicar la ecuación $\mathrm{K}=\mathrm{Wt} / \mathrm{LE}^{\mathrm{b}}$ donde: $\mathrm{Wt}=$ peso total del ejemplar $(\mathrm{g}), \mathrm{LE}=$ longitud estándar (mm), b=coeficiente angular de la regresión entre el peso total-longitud estándar.

Se realizó un análisis de componentes principales (ACP), con los valores de frecuencia de ocurrencia para cada ítem alimenticio con respecto a la estructura de talla, con el fin de estimar el porcentaje de variación de la dieta y determinar qué presas se agrupan con la estructura de tallas.

Para estimar los aspectos reproductivos se determinó la proporción de sexos (Wenner, 1972) a partir de la siguiente ecuación: \% Machos $=100 *(\mathrm{Nm} / \mathrm{Nt})$. Donde $\mathrm{Nm}=$ es el número de machos y $\mathrm{Nt}=$ el número total de individuos; se aplicó el test chi-cuadrado para identificar las diferencias estadísticas. La talla media de madurez sexual se determinó mediante el método estadístico gráfico, cuando más del $50 \%$ de la población entra en el período reproductivo (Sokal \& Rohlf, 1995). También se aplicó la relación gonadosomática (RGS) (Vazzoler, 1996), indica la relación entre el avance de la maduración ovocitaria y el peso del cuerpo, y refleja el grado de desarrollo de la gónada. Se calculó mediante la ecuación: $\mathrm{RGS}=\mathrm{Wo} / \mathrm{We} \times 100$ donde $\mathrm{Wo}=$ peso de la gónada, We=peso del cuerpo (Wt-Wo; Wt=peso total). La fecundidad (Ricker, 1968) se estimó mediante el método de las submuestras secas, que consta de un secado en horno a $40{ }^{\circ} \mathrm{C}$ durante 25 minutos, para el posterior conteo directo con una balanza analítica ADVENTURER pro av 264, hasta cuatro décimas de precisión $(0.0001 \mathrm{~g})$. Por último, se aplicó la ecuación: $\mathrm{Fa}=\Sigma \mathrm{n}^{\mathrm{o}} / \mathrm{N}^{\mathrm{o}}$, donde $\mathrm{n}^{\mathrm{o}}=$ número de ovocitos por hembra, $\mathrm{N}^{\mathrm{o}}=$ número total de hembras; con el fin de obtener el valor promedio de ovocitos en cada uno de los muestreos. El diámetro de ovocito se determinó con ayuda de papel milimetrado, mediante la alineación de todos los ovocitos secos, posibles en $10 \mathrm{~mm}$ y el número total se dividió en 10, este procedimiento se repitió tres veces para obtener un promedio por cada gónada, según el método de sub-muestras secas (Ricker, 1968), e incluye desviación estándar (D.E). 


\section{RESULTADOS}

Hábitat: El río Gaira presentó aguas claras o cristalinas, con una velocidad de corriente promedio de $0.62 \mathrm{~m} / \mathrm{s}$, su caudal promedio es de $2.3 \mathrm{~m}^{3} / \mathrm{s}$, con un mínimo de $0.5 \mathrm{~m}^{3} / \mathrm{s}$ en marzo, y un máximo en octubre de $5.2 \mathrm{~m}^{3} / \mathrm{s}$, es un río meándrico irregular; se encuentra representado en su recorrido por un sustrato rocoso y un alto número de cascadas. La temperatura ambiente y superficial del agua presentó un promedio $23.3{ }^{\circ} \mathrm{C}$ y $22.1{ }^{\circ} \mathrm{C}$ durante las bajas lluvias y $25.4{ }^{\circ} \mathrm{C}$ y $22.7{ }^{\circ} \mathrm{C}$ en altas lluvias, respectivamente. El oxígeno disuelto en general fue alto $6.6 \mathrm{mg} / \mathrm{L}$ durante las bajas lluvias, disminuye a $5.1 \mathrm{mg} / \mathrm{L}$ para la época de altas lluvias, mientras la conductividad fue de 90.2 $\mu \mathrm{s} / \mathrm{cm}$ para las bajas lluvias, y disminuye a 67.4 $\mu \mathrm{s} / \mathrm{cm}$ en altas lluvias, el $\mathrm{pH}$ tuvo tendencia a la basicidad 8.7 en bajas lluvias, disminuyen a 7.3 en altas lluvias (Cuadro 1). Sin embargo, no se observaron diferencias significativas entre las variables fisicoquímicas entre los muestreos y periodos de precipitación. En general, el sistema se observó como un ambiente oligotrófico con tendencia a la eutrofización.

Morfología del tracto digestivo: Se capturó un total de 477 individuos de $\mathrm{H}$. sierraensis. Su sistema digestivo es bien definido con una boca terminal, con labios suaves y flexibles, dientes tricúspides, estómago sacular que se ubica en la parte dorsal anterior de la cavidad abdominal, presenta en promedio seis ciegos pilóricos (cuatro a ocho), tiene un intestino corto con una longitud promedio de $52.88 \mathrm{~mm}(2.2-101.1 \mathrm{~mm})$. En general, es un sistema digestivo característico de especies carnívoras y/o insectívoras, se observó una baja y positiva correlación entre la longitud estándar y la longitud del intestino $(\mathrm{Y}=0.7147 \mathrm{x}+6.7295$, $\mathrm{r}=0.36$ ), alta, positiva y significativa correlación entre la longitud del estómago y el ancho del estómago $(\mathrm{Y}=0.5279 \mathrm{x}-0.2659, \mathrm{r}=0.61)$.

Dieta: Se examinaron 477 individuos y sus estómagos, se determinaron 32 ítems alimenticios (Cuadro 2). La especie mostró hábitos tróficos de omnívoro con tendencia a la insectivoría, la amplitud de nicho trófico fue de $\left(H^{\prime}=0.69\right)$, y es una especie eurifágica o generalista. El coeficiente de vacuidad (Va) de $1.59 \%$ equivale a siete estómagos vacíos, en altas lluvias seis y en bajas lluvias uno.

En el componente animal, la dieta estuvo constituida especialmente por larvas de insectos $(37.4 \% \mathrm{~N}, 40.5 \% \mathrm{~V}, 60.2 \% \mathrm{~F}, 4.1$ I.A), pertenecientes a Trichoptera Atanatolica sp. (0.5 I.A); seguido de Diptera, principalmente Simulium sp. (0.3 I.A); gasterópodos, con Pomacea sp. como alimento secundario (0.4 I.A); mientras que el componente vegetal estuvo conformado por material vegetal no identificado (Hojas y fibras) (6.0 I.A). Los insectos adultos Coleoptera Staphylinidae $\left(2.9 \times 10^{-3}\right.$ I.A), seguido de Chrysomelidae $\left(0.3 \times 10^{-4}\right.$ I.A)

CUADRO 1

Datos físico-químicos por períodos climáticos, en el río Gaira, Sierra Nevada de Santa Marta, Caribe, Colombia

TABLE 1

Physico-chemical data by climate period, Gaira River, Sierra Nevada of Santa Marta, Caribbean Basin, Colombia

\begin{tabular}{l|cccccc}
\multicolumn{1}{c}{ Variable } & \multicolumn{3}{c}{ Bajas lluvias } & \multicolumn{3}{c}{ Altas lluvias } \\
Color & Mínimo & Máximo & Promedio & Mínimo & Máximo & Promedio \\
Caudal $\left(\mathrm{m}^{3} / \mathrm{s}\right)$ & & Cristalino & & & Marrón \\
Temperatura ambiente $\left({ }^{\circ} \mathrm{C}\right)$ & 0.5 & 2.0 & 1.0 & 1.5 & 5.2 & 3.3 \\
Temperatura superficial del agua $\left({ }^{\circ} \mathrm{C}\right)$ & 20.4 & 25.8 & 23.3 & 20.1 & 30.7 & 25.4 \\
Oxígeno disuelto $(\mathrm{mg} / \mathrm{L})$ & 19.3 & 25.9 & 22.1 & 19.4 & 29.6 & 22.7 \\
$\mathrm{pH}$ & 5.5 & 7.8 & 6.6 & 3.6 & 6.8 & 5.1 \\
Conductividad $(\mu \mathrm{S} / \mathrm{cm})$ & 8.2 & 9.1 & 8.7 & 5.7 & 9.6 & 7.3 \\
\hline
\end{tabular}


CUADRO 2

Dieta de Hemibrycon sierraensis en el río Gaira, Sierra Nevada de Santa Marta, Caribe, Colombia

TABLE 2

Diet of Hemibrycon sierraensis, Gaira River, Sierra Nevada of Santa Marta, Caribbean Basin, Colombia

\begin{tabular}{|c|c|c|c|c|c|c|}
\hline Orden & Familia & Ítem & $\% \mathrm{~N}$ & $\% \mathrm{~V}$ & $\% \mathrm{~F}$ & I. A. \\
\hline \multirow[t]{8}{*}{ Diptera } & Psychodidae & Maurina sp. (L) & 0.43 & 0.33 & 1.06 & $2.30 \times 10^{-3}$ \\
\hline & & Clognia sp. (L) & 1.47 & 0.81 & 2.06 & 0.02 \\
\hline & Muscidae & Limnophora sp. (L) & 0.21 & 0.14 & 0.27 & $3.90 \times 10^{-3}$ \\
\hline & Blephariceridae & Limonicola sp. (L) & 0.5 & 0.45 & 1.33 & 0.01 \\
\hline & Ceratopogonidae & Allauadamyia sp. (L) & 3.46 & 1.52 & 4.97 & 0.08 \\
\hline & Simuliidae & Simulium sp. (L) & 6.79 & 3.02 & 9.35 & 0.28 \\
\hline & & Simulium sp. (P) & 1.53 & 0.9 & 1.53 & 0.01 \\
\hline & & Restos Diptera & 0 & 0.26 & 0.53 & $1.4 \times 10^{-3}$ \\
\hline \multirow[t]{2}{*}{ Hymenoptera } & Formicidae & Formicidae (A) & 2.44 & 2.87 & 4.84 & 0.14 \\
\hline & Vespidae & Vespidae (A) & 0.85 & 1.41 & 1.79 & 0.03 \\
\hline \multirow[t]{3}{*}{ Tricoptera } & Glossomatidae & Mortoniella sp. (L) & 0.35 & 0.33 & 0.86 & $2.8 \times 10^{-3}$ \\
\hline & Calamoceratidae & Banyallarga sp. (L) & 0.97 & 1.42 & 1.79 & 0.03 \\
\hline & Leptoceriidae & Atanatolica sp. (L) & 14.75 & 5.42 & 8.36 & 0.45 \\
\hline \multirow[t]{5}{*}{ Coleoptera } & Psephenidae & Psephenops sp. (L) & 0.23 & 0.44 & 0.73 & $3.2 \times 10^{-3}$ \\
\hline & Ptilodactylidae & Anchytarsus sp. (L) & 0.12 & 0.12 & 0.4 & $1 \times 10^{-3}$ \\
\hline & Staphylinidae & Staphylinidae (A) & 0.5 & 0.15 & 0.2 & $3 \times 10^{-4}$ \\
\hline & Chrysomelidae & Chrysomelidae (A) & 0.02 & 0.04 & 0.07 & $2.90 \times 10^{-4}$ \\
\hline & Elmidae & Elmidae (A) & 0.02 & 0.02 & 0.07 & $2.80 \times 10^{-5}$ \\
\hline Ephemeroptera & Baetidae & Baetodes sp. (L) & 2.57 & 1.64 & 4.11 & 0.07 \\
\hline Plecoptera & Perlidae & Anacroneunia sp. (L) & 0.12 & 0.12 & 0.4 & $5 \times 10^{-4}$ \\
\hline Megaloptera & Corydalidae & Cordalus sp. (L) & 0.02 & 0.32 & 0.07 & $2 \times 10^{-4}$ \\
\hline Odonata & Libellulidae & Libellulidae (L) & 0.06 & 0.46 & 0.2 & $9.2 \times 10^{-4}$ \\
\hline \multirow[t]{3}{*}{ Architaenioglossa } & Lymnaeidae & Lymnaea sp. & 0.39 & 0.17 & 0.8 & $1.3 \times 10^{-3}$ \\
\hline & Ampullariidae & Pomacea sp. & 8.08 & 5.76 & 7.43 & 0.43 \\
\hline & & Restos de gastropoda & 0 & 3.77 & 2.79 & 0.10 \\
\hline Microsporales & Cladophoraceae & Rhizoclonium sp. & 51.66 & 4.26 & 3.91 & 0.17 \\
\hline Sapindales & Anacardiaceae & Mangifera sp. (Fr) & 0 & 0.71 & 0.07 & $5 \times 10^{-4}$ \\
\hline \multirow[t]{5}{*}{ Otros } & & Restos vegetales & 0 & 33.85 & 17.71 & 5.99 \\
\hline & & Restos insectos & 0 & 27.77 & 19.83 & 5.51 \\
\hline & & Sustrato (rocas) & 0.81 & 0.16 & 1.13 & $1.8 \times 10^{-3}$ \\
\hline & & Ovocitos & 1.16 & 0 & 0.07 & $1.8 \times 10^{-6}$ \\
\hline & & Semilla & 0.48 & 1.36 & 1.33 & 0.02 \\
\hline
\end{tabular}

$\% \mathrm{~N}$ : porcentaje numérico, \% V: porcentaje de volumen, \% FO: porcentaje frecuencia observada, IA: Índice de importancia alimentaria, L: larva, A: adulto, P: pupa, Fr: fruto.

$\% \mathrm{~N}$ : numeric percentage, $\% \mathrm{~V}$ : percentage of volume, $\%$ FO: percentage observed frequency, IA: Index of Importance in food, L: larva, A: Adult, P: pupa, Fr: fruit.

y Elmidae (1x10-4 I.A), y el alga filamentosa Rhizoclonium sp. (0.2 I.A) fueron considerados accidentales o circunstanciales.

En bajas lluvias hubo una mayor tendencia al consumo de larvas de insectos, como Trichoptera (1.7 I.A), representado por
Atanatolica sp. (1.1 I.A), seguido de Diptera (2.4 I.A), Simulium sp. (0.5 I.A), en el período de altas lluvias hubo un aumento del consumo de Rhizoclonium sp. (0.2 I.A), seguido de material vegetal (7.3 I.A); se observaron diferencias significativas de la dieta entre 
los periodos de lluvias en volumen $(\mathrm{K}-\mathrm{W}$ V: $\left.6.26, p=1.24 \times 10^{-2}\right)$ y frecuencia de ocurrencia (K-W F: 7.30, $\mathrm{p}=0.007$ ).

Variación de la dieta entre tallas: Se estimaron cinco intervalos de tallas (I a V), escala 39.21-91.75 mm de longitud estándar (LE) y una amplitud de $10.5 \mathrm{~mm}$ entre las tallas. La talla III fue la más abundante $(60.73-71.23 \mathrm{~mm}$ LE) con 206 individuos (43.2\%), le sigue la talla II (50.22-60.72 mm LE) con $161(33.8 \%)$ y IV (71.24-81.74 mm LE) con 75 (15.7\%), la talla I (39.21-50.21 mm LE) y V (81.75-91.75 mm LE) fueron las menos abundantes con 19 $(4.0 \%)$ y $16(3.4 \%)$ respectivamente.

Entre la dieta y las estructuras de tallas se encontraron diferencias significativas en Número $\left(\mathrm{K}-\mathrm{W}, \mathrm{p}=1.91 \times 10^{-3}\right)$, Volumen $(\mathrm{K}-\mathrm{W}$, $\left.\mathrm{p}=1.67 \times 10^{-6}\right)$ y Frecuencia de Ocurrencia $\left(\mathrm{K}-\mathrm{W}, \mathrm{p}=3.67 \times 10^{-07}\right)$ de los ítems consumidos. Se observó que la talla I, no consumió Rhizoclonium sp. y tiene baja ingesta de material vegetal así como de gasterópodos, por lo tanto, se determinó a esta talla como insectívora. A medida que los individuos aumentan de tamaño (longitud estándar) se observa consumo de material vegetal (hojas y fibras); lo cual categoriza a las tallas mayores como omnívoras con tendencia insectívora.

El análisis de componentes principales (ACP) entre la dieta y la estructura de tallas, indicó que los dos primeros ejes del componente explicaron el $94 \%$ de la variación total, indica la separación entre las tallas II, III y IV en el CP1 (89.73\%) y las tallas I y V, en el CP2 $(5.04 \%)$. La talla I se agrupó con mayor consumo de larvas de Diptera tales como Maurina sp. y Limonicola sp., al igual que Coleoptera con Staphylinidae. La talla II, se asoció principalmente con el consumo de material vegetal, larvas de Diptera Allauadamyia sp. y Simullium sp., al igual que Trichoptera con Atanatolica sp. En la talla III hubo un predominio de Gastropoda Lymnaea sp. y Pomacea sp. Para la talla IV se obtuvo un agrupamiento con ítems de origen alóctono como Hymenoptera Formicidae y Vespidae; al igual que el consumo de larvas de Trichoptera Banyallarga sp. y Mortoniella sp. Finalmente, en la talla $\mathrm{V}$ se observó una tendencia generalista y fue la única talla donde se registró el consumo de adultos de Coleoptera Chrysomelidae y la larva de Megaloptera Cordalus sp. (Fig. 2).

El factor de condición K osciló entre $1.5 \mathrm{y}$ 2.5, se adecua para el crecimiento alométrico e indicó que la especie se encuentra bien alimentada. Las hembras se encuentran mejor alimentadas con respecto a los machos, en hembras el valor mínimo se registró en bajas lluvias (marzo de 2015) $(\mathrm{K}=1.5$, D.E=0.5), mientras el más alto en altas lluvias (septiembre de 2014) $(\mathrm{K}=2.5$, D.E $=0.9)$. En machos, el registro más bajo fue en altas lluvias (julio de 2014) (K= 1.3 , D.E $=0.4$ ) mientras que el registro más alto fue en altas lluvias (septiembre de 2014) $(\mathrm{K}=2.2$, D.E $=0.9)$ (Fig. 3).

Aspectos reproductivos: De los 477 ejemplares recolectados 53 estaban inmaduros por lo cual no fue posible determinar su sexo; se logró determinar el sexo a 424 , donde 241 machos (56.8 \%) y 183 hembras (43.2\%), se observó relación 1:1.3 hembra-macho con diferencias significativas $\left(\chi^{2}: 16.14, p=0.02\right)$. Las hembras dominaron en noviembre (2014) con 33 individuos $(7.8 \%)$ que coincide con el período de altas lluvias y marzo (2015) con 29 ejemplares $(6.8 \%)$ que corresponde con la época de bajas lluvias; los machos fueron más abundantes en mayo y julio (2014) con 41 (9.7 $\%)$ y $43(10.1 \%)$ ejemplares, respectivamente. La talla media de maduración sexual en hembras es de $70.6 \mathrm{~mm}$ de longitud estándar, mientras que en machos de $67.0 \mathrm{~mm}$ LE (Fig. 4).

La relación gonadosomática (RGS) en machos varía durante los períodos de lluvias; con registros mínimos durante las altas lluvias (0.00012 y 0.00024) y máximos durante las bajas lluvias $(0.00056$ y 0.00074$)$. Los valores de RGS más altos para hembras se presentaron tanto en altas (julio 2014:0.00088) como en bajas lluvias (enero: 2015:0.00141), seguidos por una disminución abrupta en altas lluvias (septiembre 2014: 0.000178742 y noviembre 2015: 0.00042819, respectivamente). Los registros bajos de RGS, para machos y hembras 


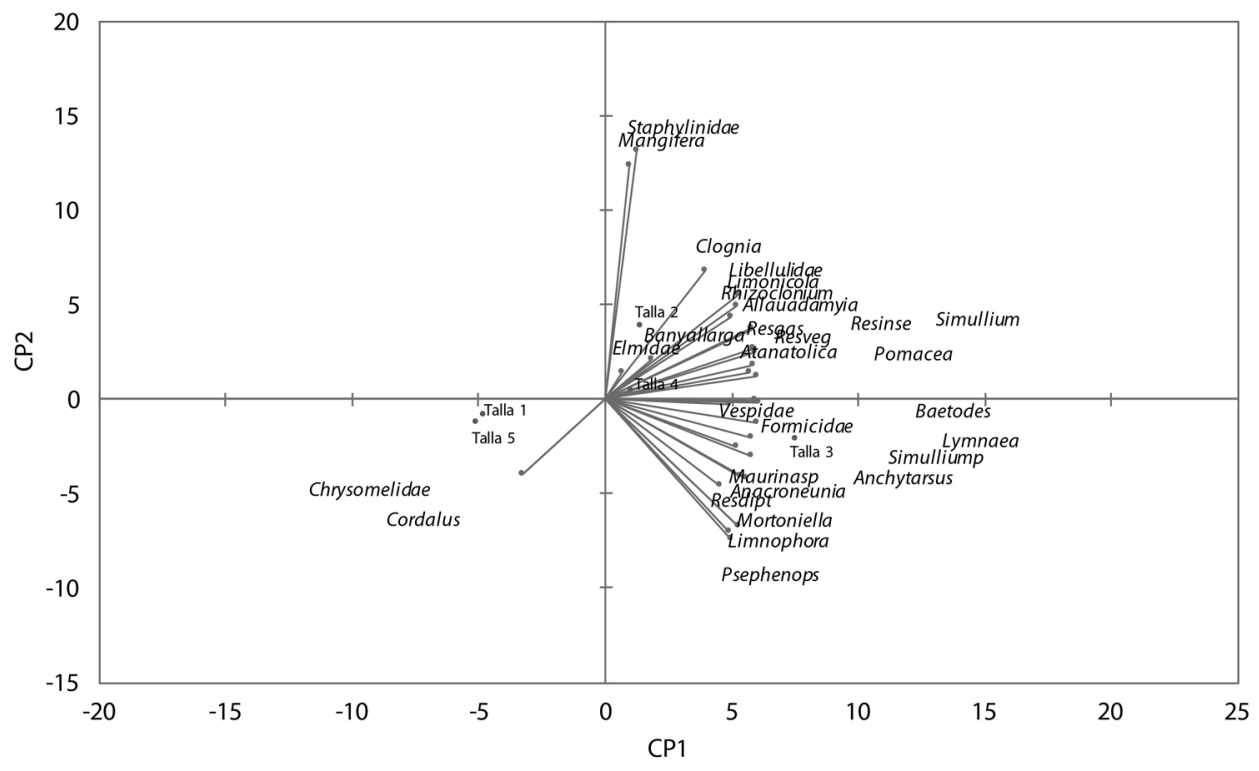

Fig. 2. Análisis de Componentes Principales (ACP) para la dieta por tallas de Hemibrycon sierraensis en el río Gaira,

Sierra Nevada de Santa Marta, Caribe, Colombia. Componente 1 en el eje Y, componente 2 en el eje X.

Fig. 2. Principal Component Analysis (PCA) of diet by length class of Hemibrycon sierraensis, in the Gaira River, Sierra

Nevada of Santa Marta, Caribe, Colombia. Component 1 on the axis X and component 2 on the axis $\mathrm{Y}$.

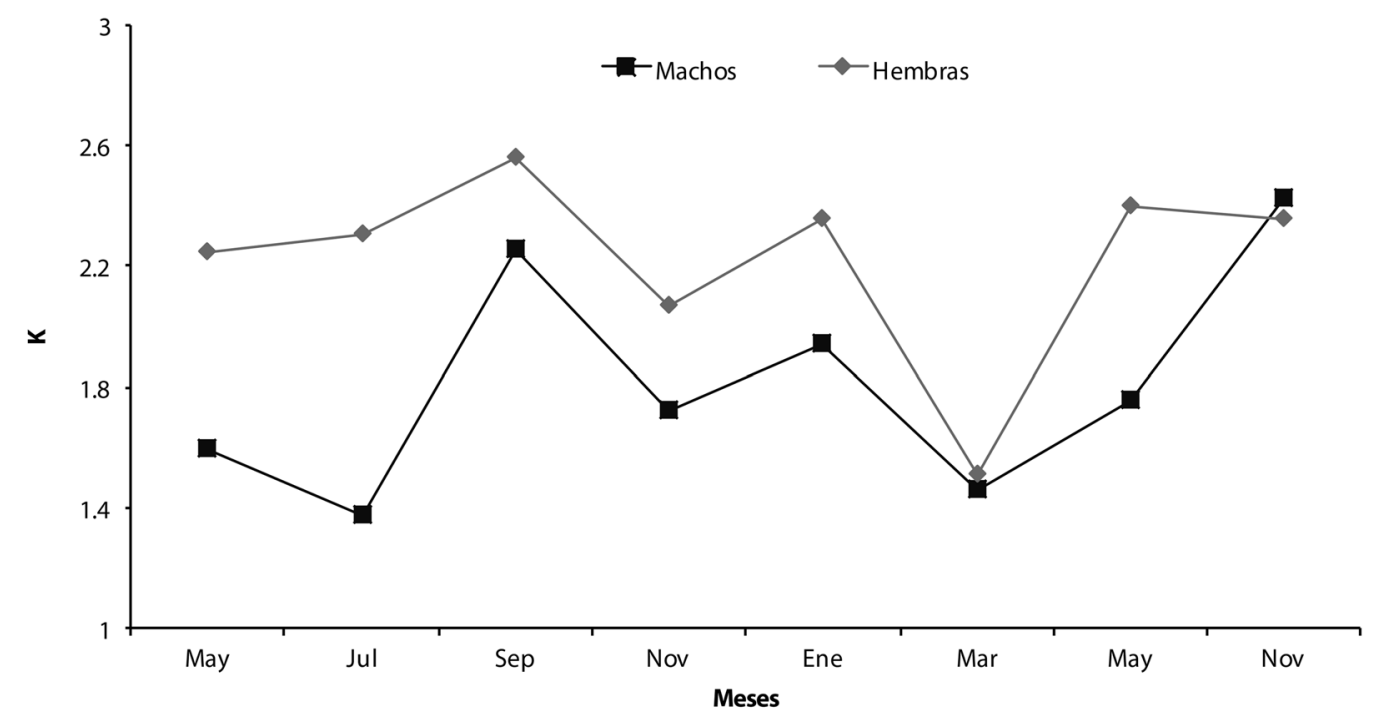

Fig. 3. Factor de condición (K) para machos y hembras de Hemibrycon sierraensis, en el río Gaira, Sierra Nevada de Santa Marta, Caribe, Colombia.

Fig. 3. Condition factor $(\mathrm{K})$ for male and female Hemibrycon sierraensis, in the Gaira River, Sierra Nevada of Santa Marta, Caribe, Colombia. 


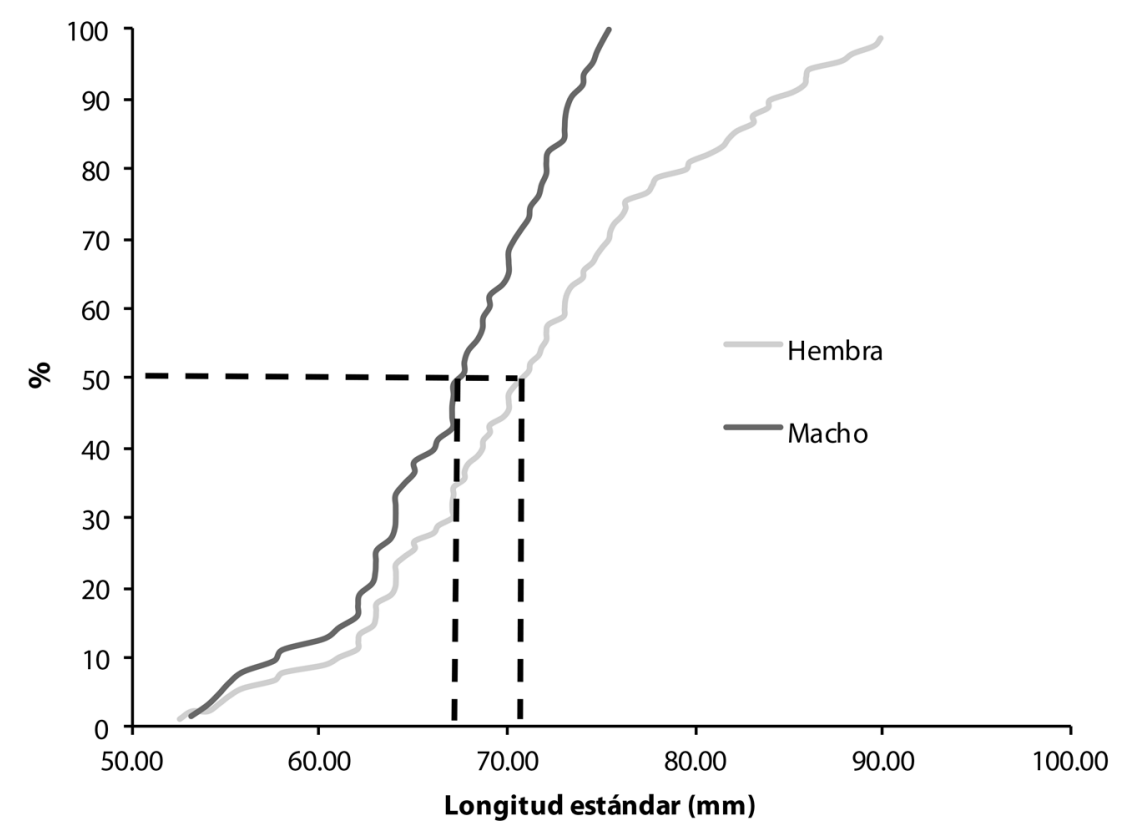

Fig. 4. Talla de madurez sexual para machos y hembras de Hemibrycon sierraensis en el río Gaira, Sierra Nevada de Santa Marta, Caribe, Colombia.

Fig. 4. Size at sexual maturity for male and female Hemibrycon sierraensis, in the Gaira River, Sierra Nevada of Santa Marta, Caribe, Colombia.

correspondieron altas lluvias (agosto-septiembre, octubre-noviembre); éstos a su vez constituyen los dos momentos en el cual la población lleva a cabo su desove (Fig. 5). No se observó relación directa entre la RGS y el factor de condición. Sobre la fecundidad, se contabilizaron 1284 ovocitos (30-4874; D.E= 882.2) en bajas y 1304 ovocitos (24-3835; D.E= 1016.9$)$ por hembra durante altas lluvias. Se observó correlación positiva entre la longitud estándar y fecundidad $(Y=39.736 x-1425.5, r=0.41)$ y entre la longitud estándar y peso de gónada $(\mathrm{Y}=0.0236 \mathrm{x}-1.1509, \mathrm{r}=0.47)$. Los ovocitos presentaron en general un diámetro de 0.07 $\mathrm{mm}(0.05-0.09 \mathrm{~mm}$; D.E $=0.01)$ para las bajas lluvias y $0.06 \mathrm{~mm}(0.05-0.09 \mathrm{~mm}$; D.E= 0.01) en altas lluvias. Se obtuvo una correlación positiva entre el oxígeno disuelto y el diámetro de ovocito $(\mathrm{Y}=0.0019 \mathrm{x}+0.0556, \mathrm{r}=0.63)$. Los ovocitos son de color amarillo claro y esféricos.

\section{DISCUSIÓN}

Para especies del género Hemibrycon, existe un patrón marcado, en el cual la dieta se basa en larvas acuáticas, así como en un alto consumo de insectos de origen alóctono (Román-Valencia \& Botero, 2006; RománValencia et al., 2008; Román-P. et al., 2014). Para Hemibrycon brevispini, especie del alto río Cauca en Colombia, se registró como una especie insectívora con una dieta dominada por Diptera, Hymenoptera y Ephemeroptera (Román-P. et al., 2014); resultados que coinciden con los reportados en este estudio donde las larvas de Diptera hacen parte de los ítems de mayor importancia dentro de la dieta de la especie, pero difieren en la abundancia, ya que Simuliidae es la familia que dominan en la dieta de $H$. sierraensis, mientras que para $H$. brevispini dominan Ceratopogonidae y Culicidae. El efecto de los períodos climáticos tiene 


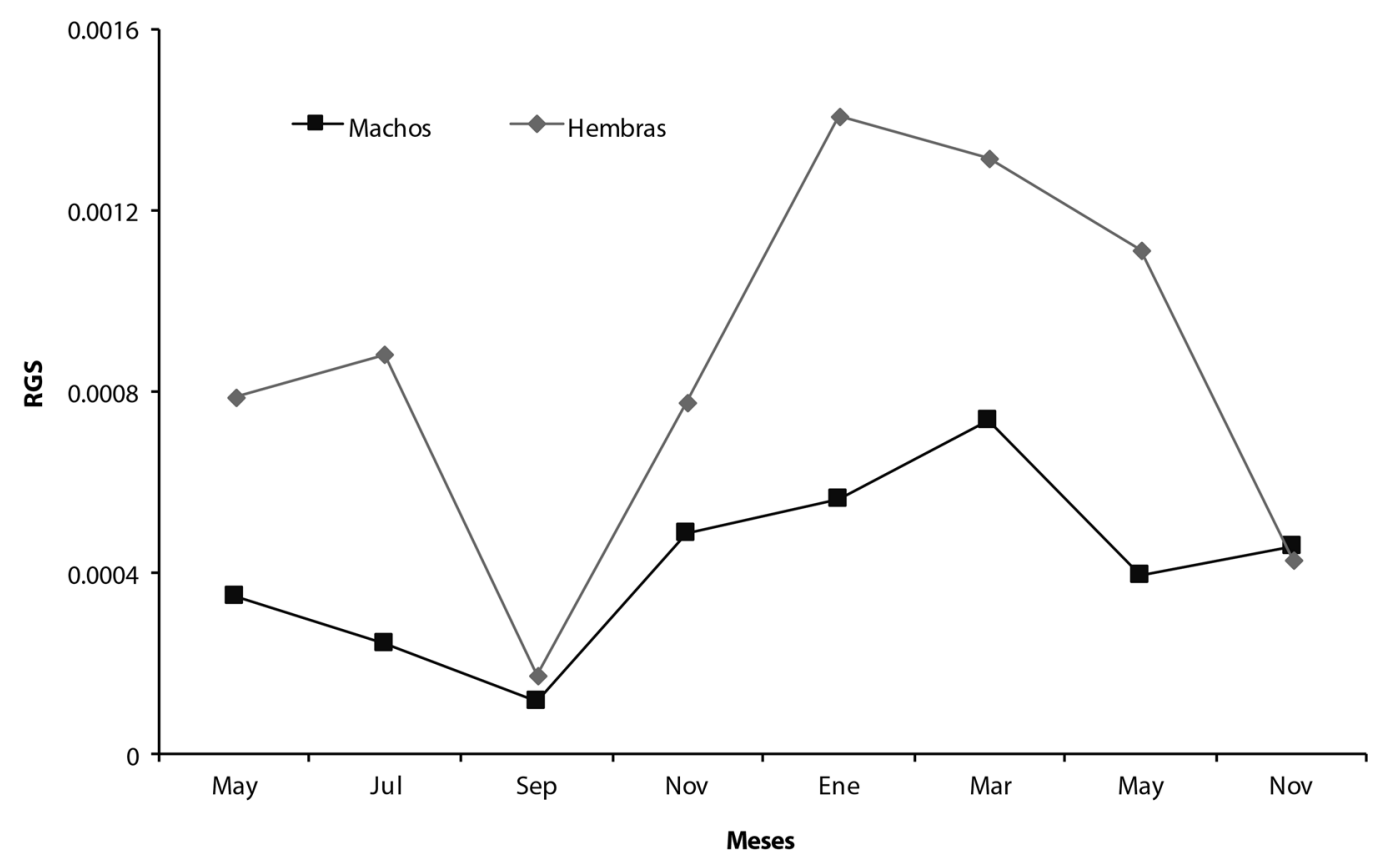

Fig. 5. Relación Gonadosomática (RGS) para machos y hembras de Hemibrycon sierraensis en el río Gaira, Sierra Nevada de Santa Marta, Caribe, Colombia.

Fig. 5. Gonasomatic relationships (RGS) for male and female Hemibrycon sierraensis, in the Gaira River, Sierra Nevada of Santa Marta, Caribe, Colombia.

como resultado un cambio en la disponibilidad del alimento (cantidad y calidad), principalmente en períodos de inundación, cuando se produce un cambio en la estructura y el sustrato del hábitat (Aranha et al., 2000; Mazzoni \& Resende, 2003; Morales \& García-Alzate, 2016). La variación de la dieta entre periodos de lluvias está relacionada con la cantidad de alimento consumido de los diferentes componentes; esto puede estar relacionado con la disponibilidad de los mismos en el ambiente, debido a que las condiciones ambientales pueden favorecer algunas presas en particular asociadas a la variaciones de nutrientes (Ortaz, Martín, \& López-Ordaz, 2011) y a los aportes de origen alóctono.

Es frecuente considerar el periodo de aguas bajas como el de menor disponibilidad de alimento para los peces invertívoros, aunque no es un patrón estricto a lo largo de un río de alta montaña, ya que depende en gran medida de otros factores como la altitud (Ortaz et al.,
2011). De hecho, en el río Gaira, RodríguezBarrios, Ospina-Tórres y Turizo-Correa (2011) encontraron que el aporte en abundancia y biomasa de macroinvertebrados acuáticos fue mayor durante las bajas lluvias y coinciden con el mayor consumo de larvas de insectos por $H$. sierraensis en este periodo. El factor de condición $\mathrm{K}$ reportó que las hembras se encuentran mejor alimentadas con respecto a los machos, y esto tiene una gran relación con la demanda energética que tienen las hembras en los procesos fisiológicos y reproductivos, registros superiores a 1.5 permiten inferir condiciones nutricionales favorables para la especie y una alta oferta alimentaria. La proporción sexual global de $H$. sierraensis fue mayor en machos con respecto a las hembras, resultado que coincide con lo reportado para dos especies sintópicas H. boquiae y Bryconamericus caucanus (Román-Valencia et al., 2008). Para H. brevispini se registra predominio de machos durante toda la fase del estudio (Román-P. et al., 2014), 
al igual que para $H$. quindos en la cual se observa la tendencia de la especie de conservar proporción mayor de machos (Román-Valencia \& Botero, 2006). Una mayor proporción de machos con respecto a las hembras, garantiza mayor cantidad de esperma para fecundar una alta cantidad de ovocitos, es de esperar una relación 1:1 en poblaciones naturales, pero distintos factores podrían generar un sesgo en el censo de una población, algunas especies segregan machos de hembras, por lo que un muestreo reducido o mal planteado puede conducir a conclusiones alejadas de la realidad (Granado-Lorencio, 2002).

$H$. sierraensis registra dos eventos reproductivos (desove) uno principal en septiembre y otro secundario en noviembre; presenta estrategia reproductiva $\mathrm{r}$ - estacional, se reproduce en sincronía con las lluvias y difieren con lo reportado para $H$. boquiae la cual se reproduce en período seco (Román-Valencia et al., 2008); de igual manera con $H$. brevispini la cual tiene tres picos reproductivos (diciembre, abril y agosto) (Román-P. et al., 2014), así como para $H$. quindos que presenta dos eventos reproductivos, en bajas y altas lluvias; lo cual indica que no existe un patrón marcado sobre el número de eventos reproductivos para las especies del género; esta variación podría estar influenciada principalmente por la interacción entre las lluvias y las condiciones ambientales particulares de cada tipo de sistema acuático quienes ofrecen alimento para los adultos y favorecen el éxito reproductivo. Por otra parte, la fecundidad de una especie es el resultado de un proceso evolutivo en el que se optimiza el reparto de energía obtenida por la alimentación entre el consumo metabólico, el crecimiento y la reproducción, $H$. sierraensis presenta fecundidad alta al comparar con otras especies de Hemibrycon: para $H$. quindos (405 ovocitos) (Román-Valencia \& Botero, 2006), H. boquiae (376 ovocitos) (Román-Valencia et al., 2008) y H. brevispini (776 ovocitos) (Román-P. et al., 2014). En especies de Characidae como Bryconamericus caucanus se reporta una fecundidad de 1965 ovocitos en promedio (Román-Valencia et al., 2008), siendo mayor a la registrada para H. sierraensis (1 284 ovocitos). Einum y Fleming (2002) discuten que el tamaño de los ovocitos depende de las variables ambientales entre estas el oxígeno disuelto y plantean que entre más alta sea la concentración de oxígeno menor es el diámetro de ovocito, este planteamiento es contrario con lo observado para H. sierraensis y podría ser explicado por la relación directa que existe entre alta fecundidad y pequeño tamaño de ovocitos, basados en que especies que maduran ovocitos de gran tamaño, requerirían mayor espacio abdominal o en su defecto la disminución del número de los mismos, y por ende de la fecundidad (Araya, Hirt, \& Flores, 2003; Thorsen, Marshall, \& Kjesbu, 2006); pero es independiente en este caso el tamaño del ovocito con las altas concentraciones de oxígeno disuelto en el agua, característica en ríos de alta montaña. De acuerdo con las características propuestas por Winemiller (1989) H. sierraensis presenta estrategia reproductiva $r$ - estacional, común en algunas especies de la familia Characidae (Winemiller, 1989), en la cual no hay cuidado parental, los individuos maduran a edades tempranas, tienen una fecundidad alta además de ovocitos de pequeño diámetro y una tasa de crecimiento elevada (Winemiller, 1989).

En 1980 la Sierra Nevada de Santa Marta, en reconocimiento al enorme valor que encierra como ecosistema natural, es declarada por la Unesco Reserva del Hombre y de la Biosfera, el corregimiento de Minca hace parte de los espacios que galardonan a la Sierra como patrimonio ambiental, la descripción de una nueva especie de pez es la prueba de que existe un alto número especies no documentadas. La sobreexplotación de los recursos locales, los residuos, la contaminación y las prácticas dañinas que se observan en el área como fogatas, preparación de alimentos y vertimientos irregulares al río, deterioran el ambiente y afectan directamente la biodiversidad allí residente. Los factores de origen antropogénico anteriormente mencionados, han afectado los sistemas acuáticos (Allan \& Flecker, 1993; Winemiller, Marrero, \& Taphorn, 1996), generan un cambio en la calidad del agua y conducen a la disminución 
significativa de poblaciones de peces (Jiménez-Segura et al., 2016). Por lo cual, se hace necesario diseñar un plan de manejo y de conservación de los recursos naturales (flora y fauna acuática y terrestre) en el área de estudio.

\section{AGRADECIMIENTOS}

Al grupo de investigación Biodiversidad del Caribe Colombiano por el préstamo de equipos. Donald C. Taphorn redactó el abstract. A la comunidad del corregimiento de Minca en SNSM por el apoyo brindado durante la fase de campo. A los integrantes del semillero de Ictiología Continental, estudiantes del Curso de Zoología III (2014 I; 2015 I) y al Instituto de Hidrología, Meteorología y Estudios Ambientales de Colombia (IDEAM).

\section{RESUMEN}

El conocimiento de los hábitos alimenticios de las especies permite evaluar su estatus en la comunidad, así como el papel funcional de un animal en su ecosistema; es decir la relación de el con todos los recursos disponibles. Se realizaron 8 muestreos bimensuales entre mayo 2014 y noviembre 2015. Para el análisis del contenido estomacal se utilizó el método numérico $(\% \mathrm{~N})$, volumen $(\% \mathrm{~V})$ y frecuencia de ocurrencia ( $\%$ FO), índice de importancia alimentaria (I.A), coeficiente de vacuidad (C.V), amplitud de nicho trófico $\left(\mathrm{H}^{\prime}\right)$ y factor condición $(\mathrm{K})$. En el análisis reproductivo se calculó la proporción sexual, la relación gonadosomática (RGS), fecundidad, talla mínima y media de madurez sexual y diámetro de ovocitos. Adicionalmente se midieron variables físicoquímicas. El río Gaira es meándrico irregular, presenta aguas claras, con altas velocidades de corriente oligotróficas con tendencia a la eutrofización. Valores de oxígeno disuelto son altos $(5.4 \mathrm{mg} / \mathrm{L})$ y conductividad eléctrica de $68.63 \mu \mathrm{S} / \mathrm{cm}$, pH neutro. Se analizaron 477 ejemplares, el coeficiente de vacuidad: $1.59 \%$, la especie presenta hábitos alimenticios eurifágicos $\left(\mathrm{H}^{\prime}=\right.$ $0.69)$, con hábitos alimenticios de omnívora con tendencia insectívora, su dieta consistió principalmente de larvas de Tricoptera donde Atanatolica sp.; seguido de Gastropoda con Pomacea sp. al igual que larvas de Diptera, siendo Simulium sp. el que más aportó en la dieta. Existen diferencias significativas entre tallas con respecto a los ítems consumidos, entre los períodos climáticos en volumen y frecuencia de ocurrencia de los ítems. El factor de condición indica que la especie estuvo bien alimentada. Se determinó el sexo de 424 ejemplares donde 241 fueron machos y 183 hembras con una proporción sexual global 1:1.3 (hembra-macho) y existieron diferencias significativas
$\left(\mathrm{Chi}^{\wedge} 2: 16.14, \mathrm{p}=0.02\right)$. La relación gonadosomática mostró que se reproduce en aguas altas, tiene una fecundidad alta con diámetro pequeño, los machos alcanzan la talla media de madurez sexual a $67.08 \mathrm{~mm}$ L.E, mientras que las hembras a los $70.68 \mathrm{~mm}$ L.E, además presenta estrategia $\mathrm{r}$ estacional. Se recomienda establecer planes de manejo y conservación de esta especie debido a la intervención de origen antrópica por el creciente turismo en la zona.

Palabras claves: dieta, reproducción, Teleósteos, diversidad, peces tropicales de agua dulce.

\section{REFERENCIAS}

Allan, J. D., \& Flecker, A. S. (1993). Biodiversity conservation in running waters. BioScience, 43, 32-43.

Aranha, J. M., Gomes, J. H., \& Fogaça, F. N. (2000). Feeding of two species of Characidium, $C$. lanei and $C$. pterostictum (Characidiinae) in a coastal stream of Atlantic Forest (Southern Brazil). Brazilian Archives of Biology and Technology, 43, 527-531.

Araya, P., Hirt, L., \& Flores, S. (2003). Biología reproductiva y crecimiento de Pimelodus clarias maculatus (Lac. 1803) (Pisces, Pimelodidae) en la zona de influencia del embalse Yacyretá. Ecología Austral, $13,83-95$.

Bellocq, M. I. (2000). A review of the trophic ecology of the Barn Owl in Argentina. Journal of Raptor Research, 34, 108-119.

Bojsen, B. H., \& Barriga, R. (2002). Effects of deforestation on fish community structure in Ecuadorian Amazon streams. Freshwater Biology, 47, 2246-2260.

Domínguez, E., \& Fernández, H. (2009). Macroinvertebrados bentónicos sudamericanos: Sistemática y biología. Tucumán, Argentina: Fundación Miguel Lillo.

Einum, S., \& Fleming, I. A. (2002). Does within-population variation in fish egg size reflect maternal influences on optimal values. The American Naturalist, 160, 756-765.

García-Alzate, C. A., Román-Valencia, C., \& Barrero, A. M. (2012). Biología alimentaria y reproductiva de Farlowella vittata (Siluriformes: Loricariidae) en la cuenca del río Güejar, Orinoquía, Colombia. Revista de Biología Tropical, 60, 1873-1888.

García-Alzate, C. A., Román-Valencia, C., \& Taphorn, D. C. (2015). A new species of Hemibrycon (Characiformes: Characidae), a freshwater fish from the Sierra Nevada of Santa Marta, Caribbean drainage, Colombia. Cuadernos de Investigación UNED, 7, 89-98.

Granado-Lorencio, C. (2002). Ecología de Peces. Sevilla: Secretariado de Publicaciones de la Universidad de Sevilla. 
Hyslop, E. J. (1980). Stomach contents analysis-a review of methods and their application. Journal of Fish Biology, 17, 411-429.

Jiménez-Segura, L. F., Galvis-Vergara, G., Cala-Cala, P., García-Alzate, C. A., López-Casas, S., Ríos-Pulgarín, M. I., ... \& Álvarez-León, R. (2016). Freshwater fish faunas, habitats and conservation challenges in the Caribbean river basins of north-western South America. Journal of Fish Biology, 89, 65-101.

Kawakami, E., \& Vazzoler, G. (1980). Método gráfico e estimativa de índice alimentar aplicado no estudo de alimentação de peixes. Boletín del Instituto Oceanográfico de Venezuela, 29, 205-207.

Krebs, C. J. (1998). Ecological Methodology (2nd ed). Menlo Park California: Addison-Welsey Educational Publisher, Inc.

Lasso, C. A., Sánchez-Duarte, P., \& Blanco-Libreros, J. F. (2015). XII. Cuencas pericontinentales de Colombia, Ecuador, Perú y Venezuela: Tipología, Biodiversidad, Servicios ecosistemicos y sostenibilidad de los ríos, quebradas y arroyos costeros. Bogotá, D.C., Colombia: Instituto de Investigación de Recursos Biológicos Alexander von Humboldt (IAvH).

Lauzanne, L. (1975). Regime alimentaire d'Hydrocyon forskalii dans le lac Tchad et ses tributaires. Hydrobiologia, 9, 105-121.

Luiz, E. A., Agostinho, A. A., Gomes, L. C., \& Hahn, N. S. (1998). Ecología trófica de peixes em dois riachos da bacia do río Paraná. Revista Brasileira de Biología, $58,273-285$.

Mazzoni, R., \& Resende, C. (2003). Seazonal diet shift a tetragonopterinae (Osteichthyes, Characidae) from the Ubatiba river, RJ, Brazil. Brazilian Journal of Biology, 63, 69-74.

Morales, J., \& García-Alzate, C. A. (2016). Estructura trófica de los peces en arroyos del Corral de San Luis, cuenca del Bajo Magdalena, Caribe, Colombia. Revista de Biología Tropical, 64, 715-732.

Ortaz, M., Martín, R., \& López-Ordaz, A. (2011). Variación espacial y temporal en la composición de la dieta de peces invertívoros en un río neotropical, Venezuela. Revista de Biología Tropical, 59, 1217-1231.

Ricker, W. E. (1968). Methods for assessment of fish production in fresh waters. Oxford: Blackwell Scientific.

Rodríguez-Barrios, J., Ospina-Tórres, R., \& TurizoCorrea, R. (2011). Grupos funcionales alimentarios de macroinvertebrados acuáticos en el río
Gaira, Colombia. Revista de Biología Tropical, 59, 1537-1552.

Roldan, G. (1988). Guía para el estudio de los macroinvertebrados acuáticos del Departamento de Antioquia. Bogotá: Impreades Presencia S.A. Colombia.

Román, P. C., Román-Valencia, C., \& Taphorn, D. C. (2014). Trophic and reproductive ecology of a neotropical characid fish Hemibrycon brevispini (Teleostei: Characiformes). Caldasia, 36, 289-304.

Román-Valencia, C., \& Botero, A. (2006). Trophic and reproductive ecology of a species of Hemibrycon (Pisces: Characidae) in Tinajas creek, Quindío River drainage, upper Cauca basin, Colombia. Revista del Museo Argentino de Ciencias Naturales, Nueva Serie, 8, 1-8.

Román-Valencia, C., Ruiz, R., \& Giraldo, A. (2008). Dieta y reproducción de dos especies sintópicas: Hemibrycon boquiae y Bryconamericus caucanus (Pisces: Characidae) en la quebrada Boquía, Río Quindío, Alto Cauca, Colombia. Revista del Museo Argentino de Ciencias Naturales, Nueva Serie, 10, 55-62.

Shannon, C. E., \& Weaver, W. (1949). The mathematical theory of communication. Champaign: University of Illinois Press.

Sokal, R. R., \& F. J. Rohlf. (1995). Biometry. San Francisco: W.H. Freemand.

Streble, H., \& Krauter, D. (1987). Atlas de los microorganismos de agua dulce: La vida en una gota de agua. Barcelona: Omega.

Thorsen, A., Marshall, C. T., \& Kjesbu, O. S. (2006). Comparison of various potential fecundity models for north-east Arctic cod Gadus morhua, L. using oocyte diameter as a standardizing factor. Journal of Fish Biology, 69, 1709-1730.

Vazzoler, A. E. (1996). Biología da reproduçâo de peixes teleósteos: teoría e práctica. São Paulo: EDUEM.

Wenner, A. M. (1972). Sex ratio as a function of size in marine Crustacea. American Naturalist, 106, 321-350.

Winemiller, K. O. (1989). Patterns of variation in life history among South American fishes in seasonal environments. Oecologia, 81, 225-241.

Winemiller, K. O., Marrero, C., \& Taphorn, D. C. (1996). Perturbaciones causadas por el hombre a las poblaciones de peces de los llanos y del piedemonte Andino de Venezuela. Biollania, 12, 13-48. 